\title{
Subtle Upper Limb Impairment in Radiological Isolated Syndrome Subjects
}

\author{
Solaro $\mathrm{C}^{1^{*}}$, Trabucco $\mathrm{E}^{1}$, and $\mathrm{M}$ Messmer Uccelli ${ }^{2}$ \\ ${ }^{1}$ Neurology Unit, Department of Head and Neck, ASL3 Genovese Genova, Italy \\ ${ }^{2}$ Department of Social and Health Research, AISM Italian Multiple Sclerosis Society, Genova, Italy
}

*Corresponding author: Solaro C, Neurology Unit, Department of Head-Neck, ASL3 Genovese Genova Largo Rosso 3 Genova 16153 Italy, Tel: + 390108498365 ; Fax: + 39010 8498046; E-mail: csolaro@libero.it

Rec date: May 02, 2014, Acc date: Jul 23, 2014, Pub date: Jul 26, 2014

Copyright: ( 2014 Solaro C, et al. This is an open-access article distributed under the terms of the Creative Commons Attribution License, which permits unrestricted use, distribution, and reproduction in any medium, provided the original author and source are credited.

\begin{abstract}
Objective: Spatial dissemination of focal white matter brain lesions, highly suggestive of multiple sclerosis (MS) in subjects without clinical neurological symptoms, is referred to as radiological isolated syndrome (RIS).

The aim of this study was to demonstrate that kinematic parameters are able to measure motor performance and to identify subtle symptoms undetectable with the clinical examination.

Methods: We investigated the clinical, kinematic motor performance in 6 subjects following conventional brain MRI (1.5-T). Subjects had a negative neurological examination and fulfilled the diagnostic criteria for RIS proposed by Okuda.

Results: Subjects were 4 females and 2 males with a mean age of 39.5 years. 2 subjects performed all kinematic parameters in the range of $\mathrm{HC} / \mathrm{M}, 1$ subject resulted with one parameter above the range of $\mathrm{HC} / \mathrm{M}$ group, 3 subjects had more than 2 kinematic parameters above the range of HC/M. The mean nine-hole peg test (9HPT) score was 18 seconds \pm 1.2 subjects had spinal cervical cord lesions and oligoclonal bands were detected in other 2 patients. VEP were negative in all subjects. No case showed dissemination in time (no new T2 lesions at the 6 month MRI). Of the kinematic parameters used, the integral of the norm of the third derivative-jerk-of the hand path was the most sensitive parameter (four of six subjects), Remarkably the technique appears to be able to detect subtle differences between some subjects with RIS and control subjects.
\end{abstract}

Conclusion: We believe that this type of instrumental assessment can help in identifying subjects with RIS with a high risk for developing MS.

Keywords: RIS; Kinematic analyses; Multiple sclerosis

\section{Introduction}

Spatial dissemination of focal white matter brain lesions, highly suggestive of multiple sclerosis (MS) in subjects without clinical neurological symptoms, is referred to as radiological isolated syndrome (RIS) [1]. The natural course of RIS is largely unknown and the clinical conversion to clinically isolated syndrome (CIS) or MS is related to the presence of spinal cord lesions (positive predictive value around 90\%) [2].

A recent MRI spectroscopy study showed that NAA/Cr levels were at least 2 SDs below the healthy control mean in $44 \%$ of RIS subjects in normal-appearing white matter and in the grey matter of $61 \%$ RIS subjects [3]. Cognitive evaluation showed impairment in eight out of 29 RIS subjects [4]. These reports underline the complexity of classifying RIS subjects.

We have previously used kinematic analysis to demonstrate suitable motor changes in upper limb function in asymptomatic CIS suggestive of MS [5]. The hypothesis is that although the clinical examination is normal, some motor tasks may be impaired due to lesions in the central nervous system. We investigated the clinical, kinematic motor performance in six subjects following conventional brain MRI (1.5-T),
(Table 1). Subjects had a negative neurological examination and fulfilled the diagnostic criteria for RIS proposed by Okuda [1]. White matter abnormalities on brain MR satisfied Barkhof s criteria (at least three out of 4 criteria) for dissemination in space. Subjects originally underwent MRI for tension headache (three subjects), trauma (one subject) and transient paresthesia (two subjects). Subjects were four females and two males with a mean age of 39.5 years. Other pathologic conditions were ruled out based on symptom history and appropriate laboratory testing. A second MRI scan was performed six months after the original scan. RIS subjects and controls were not in treatment with benzodiazepine, antiepileptic medications or B-blockes. Subjects underwent a lumbar puncture and visual evoked potentials. Kinematic data were compared to six gender- and age-matched healthy controls and subjects with migraine.

\begin{tabular}{|l|l|l|l|l|l|l|l|}
\hline $\begin{array}{l}\text { Subject } \\
\text { number }\end{array}$ & Gender & Age & $\begin{array}{l}\text { Clinical } \\
\text { presentation }\end{array}$ & $\begin{array}{l}\text { Spinal } \\
\text { Cord } \\
\text { MRI }\end{array}$ & $\begin{array}{l}\text { CSF- } \\
\text { OB }\end{array}$ & VEP & $\begin{array}{l}\text { Number } \\
\text { of } \\
\text { positive } \\
\text { KP }\end{array}$ \\
\hline 1 & F & 34 & H & - & + & - & 0 \\
\hline 2 & F & 22 & HT & - & - & - & 0 \\
\hline 3 & F & 42 & H & - & - & - & 1 \\
\hline
\end{tabular}


Page 2 of 2

\begin{tabular}{|l|l|l|l|l|l|l|l|}
\hline 4 & M & 44 & H & + & - & - & $>2$ \\
\hline 5 & M & 48 & P & + & + & - & $>2$ \\
\hline 6 & F & 46 & P & - & - & - & $>2$ \\
\hline
\end{tabular}

Table 1: Motor performance in six subjects following conventional brain MRI

MRI: Magnetic Resonance Imaging; H: Headache; T: Head Trauma; P: Paresthesia; CSF: Cerebrospinal Fluid; OB: Oligoclonal Bands; VEP: Visual Evoked Potential; KP: Kinematic Parameters.

The experimental design, kinematic protocol and kinematic indicators are described elsewhere [5]. Briefly, subjects were instructed to perform planar, center-out reaching toward targets, presented on a 17 " LCD screen, on the horizontal plane of a digitizing tablet (Drawing Board III, CalComp, USA), by means of a cordless transducer in the form of a pen. This was performed in 8 different directions. The experimental protocol for each session included two blocks of trials; movements toward each direction were repeated five times, for a total of 40 trajectories for each block and subjects performed the experiment with both left and right hands successively. A number of quantitative indicators of sensorimotor performance were computed, including: aiming error: the difference between target and actual initial trajectory direction (first $100 \mathrm{~ms}$ of movement); path curvature: the percent of increase of the length of the actual hand path, relative to the straight line connecting initial and target position; symmetry: the ratio between the duration of the acceleration and deceleration phases; smoothness: logarithm of the integral of the norm of the third derivative-jerk-of the hand path.

Two subjects performed all kinematic parameters in the range of $\mathrm{HC} / \mathrm{M}$, one subject (subject n. 3) resulted with one parameter above the range of $\mathrm{HC} / \mathrm{M}$ group, three subjects (subjects n. 4-6) had more than two kinematic parameters above the range of $\mathrm{HC} / \mathrm{M}$. The mean nine-hole peg test (9HPT) scores (average of four trials for each hand) was 18 seconds \pm 1 , within the normal range. Subjects $n$. 4 and 5 had spinal cervical cord lesions, while oligoclonal bands were detected in subjects $n .1$ and 5. VEP were negative in all subjects. No case showed dissemination in time, meaning no new T2 lesions at the 6 month MRI.

Of the kinematic parameters used, the integral of the norm of the third derivative-jerk-of the hand path was the most sensitive parameter (four of six subjects), as observed in our previous study on subjects with CIS. Remarkably the technique appears to be able to detect subtle differences between some subjects with RIS and control subjects. It is also relevant that this method provides quantitative data on motor performance that can also be useful in follow-up studies. We believe that this type of instrumental assessment able to measure motor performance can identified subtle symptoms undetectable with the clinical examination and can help in identifying subjects with RIS with a high risk for developing MS. It is important to note that currently it is not possible to conclude that kinematic analysis predicts clinical evolution that would assist clinicians in correctly classifying subjects with white matter lesions on MR. Longitudinal data from a large sample is warranted.

\section{References}

1. Okuda DT, Mowry EM, Beheshtian A, Waubant E, Baranzini SE, et al. (2009) Incidental MRI anomalies suggestive of multiple sclerosis: the radiologically isolated syndrome. Neurology 72: 800-805.

2. Okuda DT, Mowry EM, Cree BA, Crabtree EC, Goodin DS, et al. (2011) Asymptomatic spinal cord lesions predict disease progression in radiologically isolated syndrome. Neurology 76: 686-692.

3. Stromillo ML, Giorgio A, Rossi F, Battaglini M, Hakiki B, et al. (2013) Brain metabolic changes suggestive of axonal damage in radiologically isolated syndrome. Neurology 80: 2090-2094.

4. Amato MP, Hakiki B, Goretti B, Rossi F, Stromillo ML, et al. (2012) Association of MRI metrics and cognitive impairment in radiologically isolated syndromes. Neurology 78: 309-314.

5. Solaro C, Brichetto G, Casadio M, Roccatagliata L, Ruggiu P, et al. (2007) Subtle upper limb impairment in asymptomatic multiple sclerosis subjects. Mult Scler 13: 428-432. 